\title{
Expanding the Scope of Instant Messaging with Bidirectional Haptic Communication
}

\author{
Youngjae Kim and Minsoo Hahn \\ Korea Advanced Institute of Science and Technology \\ Korea, Republic of
}

\section{Introduction}

For the past five years, haptic interfaces have been applied to various commercial products. Most consumers are now familiar with the term haptic. Many among them use vibro-tactile feedback equipped touchscreen devices, although they may not have a clear understanding of what it is. According to the Google Trend result (http://www.google.com/trends/), Korean people type in and search for the keyword haptic more frequently than people in other countries. The traffic gaps between Korea and other countries are as follows.

\begin{tabular}{|l|r|r|l|l|r|r|}
\hline Region & \multicolumn{1}{|l|}{ Traffic } & std error & & City & Traffic & std error \\
\hline South Korea & 1 & $0 \%$ & & Seoul (South Korea) & 1 & $0 \%$ \\
\hline Vietnam & 0.475 & $5 \%$ & & Singapore (Singapore) & 0.435 & $5 \%$ \\
\hline Singapore & 0.395 & $5 \%$ & & Jakarta (Indonesia) & 0.22 & $10 \%$ \\
\hline Malaysia & 0.25 & $5 \%$ & & Ottawa (Canada) & 0.21 & $10 \%$ \\
\hline Philippines & 0.23 & $5 \%$ & & Bangkok (Thailand) & 0.2 & $10 \%$ \\
\hline Thailand & 0.195 & $5 \%$ & & Hong Kong (Hong Kong) & 0.175 & $10 \%$ \\
\hline Indonesia & 0.18 & $10 \%$ & & Delhi (India) & 0.115 & $10 \%$ \\
\hline Hong Kong & 0.18 & $10 \%$ & & Seattle (USA) & 0.115 & $10 \%$ \\
\hline Taiwan & 0.145 & $5 \%$ & & San Francisco (USA) & 0.115 & $10 \%$ \\
\hline India & 0.14 & $5 \%$ & & Los Angeles (USA) & 0.11 & $10 \%$ \\
\hline
\end{tabular}

Table 1. Google Trend result on the keyword haptic (data acquired on Aug. 31, 2009)

In Table 1, the numbers in the Traffic column represent the relative values calculated upon the most dominant region (in this case, South Korea). As can be seen in Table 1, the search traffic of South Korea is twice higher than those of other countries such as Vietnam, Singapore, and the USA. It is mainly due to the marketing strategy of local cellular phone manufacturers that included the term haptic in their product names. The important point is not only that people are becoming familiar with the keyword, but also that many research and industry fields are starting to focus on haptic and its effects. For example, a car manufacturer may try to apply a haptic interface to the navigation controller, or a bank may introduce ATM's with a newly installed haptic feedback-equipped touchscreen. In short, haptic technology is making gradual changes in our daily lifestyle. 
The initial goal of haptic technology is to facilitate the manipulation of devices. A vibro-tactile feedback enables a user to control a device more accurately and easily. For the next step, haptic aims to give intuitiveness to control target devices. This is mainly because, from a cognitive point of view, users expect a kind of reaction if he or she tries to command to the target.

Haptic technologies are widely employed in many areas these days, but in this chapter, we will focus on its communication usage only. As shown in many studies, haptic can be a type of daily messaging behaviours. Computer-mediated messaging technologies continue to evolve rapidly, and various types of messaging services are being marketed including short message services (SMS's) provided on a cellular phone, message-oriented networking services such as Twitter (Java et al. 2007), blogs with trackback and reply systems, and instant messenger applications that enable peer-to-peer communication in real-time. More innovative types of messaging will continue to emerge (Poupyrev, Nashida, and Okabe 2007). Regardless of the type of messaging, all services share a common goal of diversifying communications among people (Vilhjálmsson 2003). This study aims to improve messaging experiences more realistic by adding a framework for haptic interaction.

The term haptic means pertaining to the sense of touch, and thus haptic communication can be described as "communicating via touching". Bonanni had an insight into this concept and tried to implement it (Bonanni et al. 2006). He had studied the way to convey sensations from peer to peer. Rovers had introduced the vibro-tactile-pattern-embedded emoticon named HIM (A. F. Rovers and Van Essen 2004). His research method is quite similar to that proposed in this chapter. The vibro-tactile pattern is embedded into an emoticon so that users can feel more realistic sensations while engaged in instant messaging. VibeTonz (Immersion Corp 2007) is a commercialized vibro-tactile composer from Immersion. As a cellular phone with a touch screen or a conductive switch is being produced by a number of manufacturers these days, Immersion's VibeTonz technology is actively employed. VibeTonz can compose tactile output patterns along with a timeline. Although many researches led to touch-enabled emoticons (Chang et al. 2002; L. Rovers and Van Essen 2004; Aleven et al. 2006), most of these researches were limited to conveying vibro-tactile actuation. The component of touch and related sensations encompass not only tactile stimulus, but also temperature, sound, etc. For this reason, a framework to send and to receive the whole spectrum of haptic is strongly required. The objective of this research is to facilitate haptic communications among users and expand the scope of the computer-mediated conversation.

The bidirectional haptic means that a sensor and an actuator can be manipulated on a single framework. This is a simple concept, but most researches tend to focus on one side only. To achieve true haptic communication, a system providing both a sensor and an actuator within a single framework is needed. Brave introduced in-Touch (Brave and Dahley 1997) to synchronize each cylinder-like device. Two devices are connected and have both a sensor and an actuator in one single tangible object. When one user rolls one device, the motor in the other part starts to run. HAML (El-Far et al. 2006) is a haptic markup language which centers on the haptic description. This is a technical specification that tries to elevate to the MPEG standards. In this research, the Phantom device is mainly applied. HAMLET (Mohamad Eid et al. 2008) is a HAML-based authoring tool. Both HAMLET and this research aim to accomplish simplicity and efficiency in utilizing haptic for non-programmer developers and artists. However, our target users are rather general users than those of HAMLET, who uses the instant messenger as a daily communication tool. From the view of the description language, or the markup language, SensorML (Botts and Robin 2007) is one 
of the specifications to describe a sensor. The object of this markup language is to provide the sensor information as detailed as possible including the manufacturer, hardware specifications, the data type to acquire a result, etc. It can be adopted into our work, but we concluded it is too verbose to apply this SensorML to our work.

In this study, TouchCon, a next-generation emoticon for haptic-embedded communication, is proposed. The architecture of the framework to represent haptic expressions in our daily messaging and chatting is also provided. In addition, included is the hardware specially designed for testing and the summary of user preference surveys with reference to the previous researches (Kim et al. 2009; Kim et al. 2009; Shin et al. 2007).

\section{A Platform for Managing Haptic Communication}

\subsection{Overall Description}

The proposed system enables a user to manipulate haptic interaction and to share it with others. To achieve this goal, we need to summarize the requirements of the system. The system needs to support haptic actuator control, sensor data acquisition, linkage with various applications, library management, etc. One important goal of this study is to resolve the haptic expression even when two devices are not identical.

For this reason, the haptic communication framework has been designed to achieve the flexibility and the scalability. The flexibility allows the framework to invite and to manipulate different devices. To support haptic-enabled hardwares, the framework must be capable of providing a standardized gateway. Thus, the architecture adopted here has a similar goal to the middleware system (Baldauf, Dustdar, and Rosenberg 2007) from the architectural point of view. The scalability means, the framework is extensible to adopt various sensors and actuators according to their descriptions. For that, the framework has to allow various protocols. Figure 1 shows the overall architecture of the platform.

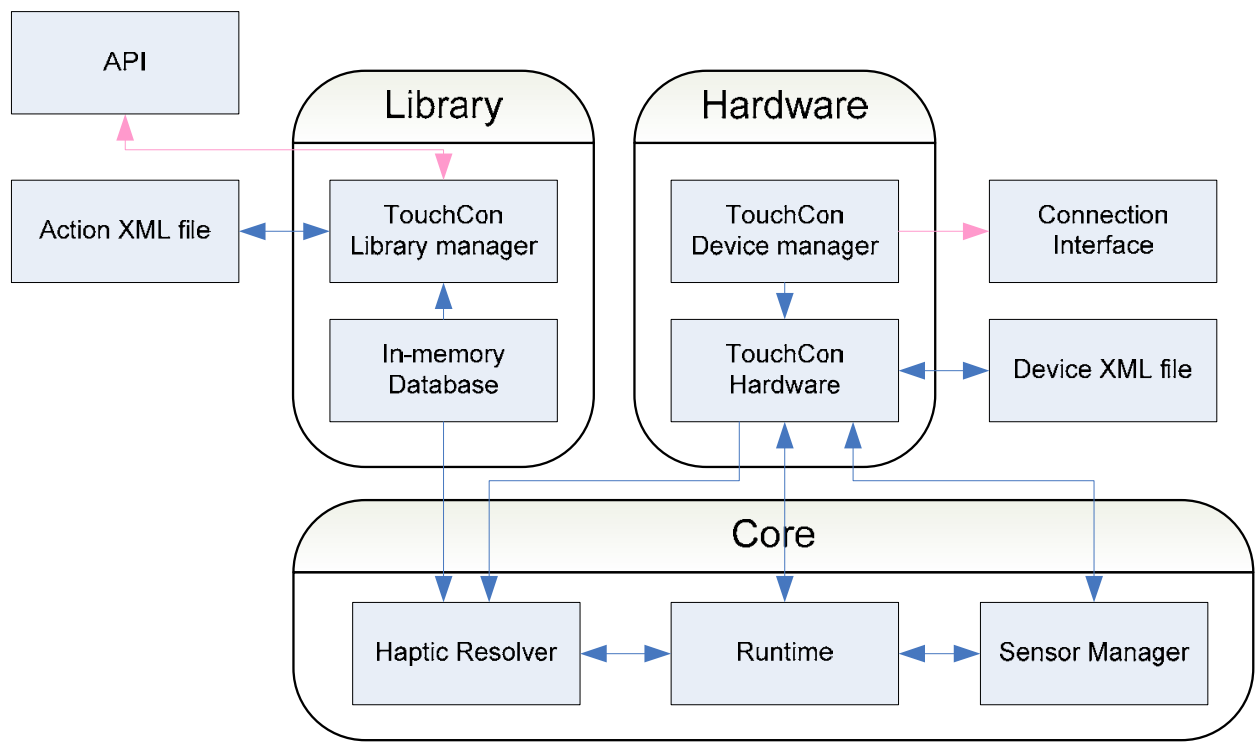

Fig. 1. Overall TouchCon architecture 
The platform consists of three main parts; the core, the library, and the hardware. The core is a runtime to execute haptic interactions. The library handles haptic emoticons and their patterns, and the hardware deals with controllable hardwares. Before moving on to elaborate on each component, it must be explained that an action stands for a single motion of haptic.

Each module is described in Table 2.

\begin{tabular}{|l|l|}
\hline Component Name & Description \\
\hline TouchCon Core & Runtime of the framework \\
\hline TouchCon Library & $\begin{array}{l}\text { A list of TouchCons and a TouchCon, which is composed by a user or } \\
\text { a haptic emoticon distributor }\end{array}$ \\
\hline TouchCon Device & $\begin{array}{l}\text { A hardware management of a TouchCon device (generally an actuator } \\
\text { or a sensor) }\end{array}$ \\
\hline Library XML file & An XML file which stores composed TouchCons \\
\hline Device XML file & $\begin{array}{l}\text { An XML file which stores hardware protocol specifications and } \\
\text { acceptable commands }\end{array}$ \\
\hline Connection Interface & Methods of communication through TouchCon hardware. \\
\hline
\end{tabular}

Table 2. Component description

To ensure the flexibility, we discriminate the library from the hardware at first. This allows for the framework to actuate similar haptic expressions with different hardware specifications. For example, there is only one red-coloured LED in the current hardware, the received TouchCon action could request to actuate the vibration motor. In this case, the resolver needs to interpret the TouchCon action into similar haptic expressions with current hardware specifications. In architectural point of view, if the hardware is directly coupled with the library and able to activate the identical hardware only, haptic expressions are limited to the hardware. To address this problem, the core runtime activates a function, i.e., the resolver, that interprets haptic expressions in accordance with hardware functionalities. The hardware monitors each available sensor and actuator so that the library acquires needed information to utilize them. For this reason, hardware management is relatively simpler than that of the library and the core in the framework.

\subsection{TouchCon Core}

The TouchCon Core consists of three components; the runtime to execute the library, the resolver to support different hardwares, and the sensor manager. The runtime module commands each haptic action to the hardware at every millisecond. In other words, a haptic action can be controlled in one millisecond. The runtime acts one of three behaviors with given TouchCon; activate user's hardware, transmit a TouchCon to a peer, or do nothing.

The resolver component modifies the input haptic action command when the hardware mismatch occurs. In other words, it compromises current hardware specifications and the input haptic expressions. Thanks to this resolver, the library can send TouchCon actions to the hardware as suitable as possible regardless of the type of the hardware attached to the user's device. The details of the resolver are given in Section 4.2.

The sensor manager processes the sensor input data. Unlike a general hardware management approach, the sensor management is done by the TouchCon Core. The reason why the sensor 
is considered as the core component and not as the hardware one is that a sensor requires to process the acquired data. For example, the user can send a 'smile' haptic action as his/her laughing sound. Namely, the microphone can act as an input sensor to the framework and this is one of the useful scenarios in our work. In short, the input expression needs a decision to be described and sent as a TouchCon action format.

\subsection{TouchCon Library}

The TouchCon Library is a bundle of TouchCon actions. It can have one or more TouchCons according to the haptic expression. The library consists of three components; the TouchCon Library manager for organizing TouchCons, the in-memory database for storing temporary TouchCon actions, and the API (Application Programming Interface) for upper level applications. The TouchCon Library manager includes an XML parser to encode and to decode the given TouchCons with the TouchCon Library schema. Since all data handled in our work are designed to use the XML only, haptic contents can be authored with no length limitation. The specification of the schema and its example are given in the next section. The API allows external applications such as an instant messenger or the internet browser to communicate with the haptic framework. Unlike commonly used API approaches, our work is coupled with hardwares. For this reason, the API restricts to be invoked by one application only. If this restriction does not exist, the hardware might be collided by commands from multiple applications.

\subsection{TouchCon Hardware}

Since the scope of this study is not restricted to the vibro-tactile actuation, the hardware component can invite different protocols. Moreover, as haptic-enabled hardwares are being produced by various manufacturers, the framework should have a room to support them. If these future changes are not taken into consideration and thus only the limited haptic expressions can be executable, the results of this study may not be applicable in the near future. One of the possible solutions is to adopt an abstract layer above the hardware driver layer, and to simplify the hardware types and the commands. These approaches are used in Microsoft Windows HAL (Hardware Abstraction Layer) architecture and JINI home network one(Arnold et al. 1999; Russinovich and Solomon 2005). Once the hardware is attached to the framework, the abstract layer loads small description files and organizes available functionalities. In general, the hardware description files are located in the web or a local system. The advantage of this approach is to provide unified control points to other applications and to enable to invite various types of haptic-enabled hardwares.

Same approach is applied to our work. Once the device is connected and the description file, we call TouchCon Device XML, is loaded successfully, the TouchCon Device Manager expects the runtime to give some commands.

\section{Haptic Description Language}

We design two haptic description XML schemas in order to manage haptic commands and to activate haptic-enabled hardwares. Three factors must be taken into consideration to design schemas. 
- Scalability: To include an abundance of haptic interactions and to support a combination of sensors and actuators, scalability must be considered in the system. This is the main reason why the XML format is adopted in this study.

- Flexibility: In this study, flexibility stands for adaptability. This means the schema can describe any form of the hardware interface. To incorporated with the framework, the developer must follow the suggested guidelines, but the developer's effort for the adaptation is minimized.

- Readability: According to Norman (Norman 2002), intuitiveness is an important factor in modern technology. From the view of consumer products, intuitiveness means easy-tounderstand, easy-to-manipulate, and easy-to-use. Likewise, the schemas in this study have been carefully designed to be understood by general users as easy as possible. For example, the SensorML schemas that describe hardware specifications tend to be highly complicated because these formats are made to achieve more complex goals; to describe every kind of sensors in full details. Besides, our schemas require to describe the basic profile, the command list, and the data type only.

\subsection{XML Schema for Haptic Device Description}

As we introduced in Section 2.4, the objective of the device description is to incorporate various types of haptic-enabled hardwares together. To ensure the bidirectional haptic communication, both the sensor and the actuator must be described in a single schema. The method we use is to put the 'Output' attribute to each device description. The 'Output' attribute is allocated as a Boolean data type. If it sets to True, it indicates an actuator. Otherwise, it is a sensor. Even though the framework separates the sensor manager from the device manager (see Figure 1), the combination of sensors and hardwares in a schema is reasonable in the sense of bidirectional haptic. The details of the TouchCon device schema are summarized in Table 3. Note that the word 'TCon' is an abbreviation of TouchCon. As can be seen in this table, we designed it with less mandatory attributes.

\begin{tabular}{|l|l|}
\hline Name & Attributes \\
\hline TConDevices & $\begin{array}{l}\text { (optional) } \\
\text { Description: specifications or vendor information }\end{array}$ \\
\hline TConDevice & (mandatory) \\
& $\begin{array}{l}\text { Name: name of the controllable device } \\
\text { Output: Boolean value for indicating sensor or actuator } \\
\text { DataType: Property for protocol } \\
\text { (optional) } \\
\text { Description: information of the component }\end{array}$ \\
\hline Property & (mandatory) \\
& Name: name to be displayed on the component \\
& Start: Start command \\
End: End command
\end{tabular}

Table 3. Description on the haptic device schema

As can be seen in Table 3, we designed it with less mandatory attributes. Note that the word TCon is an abbreviation of TouchCon. The example using the schema is in Figure 2. 


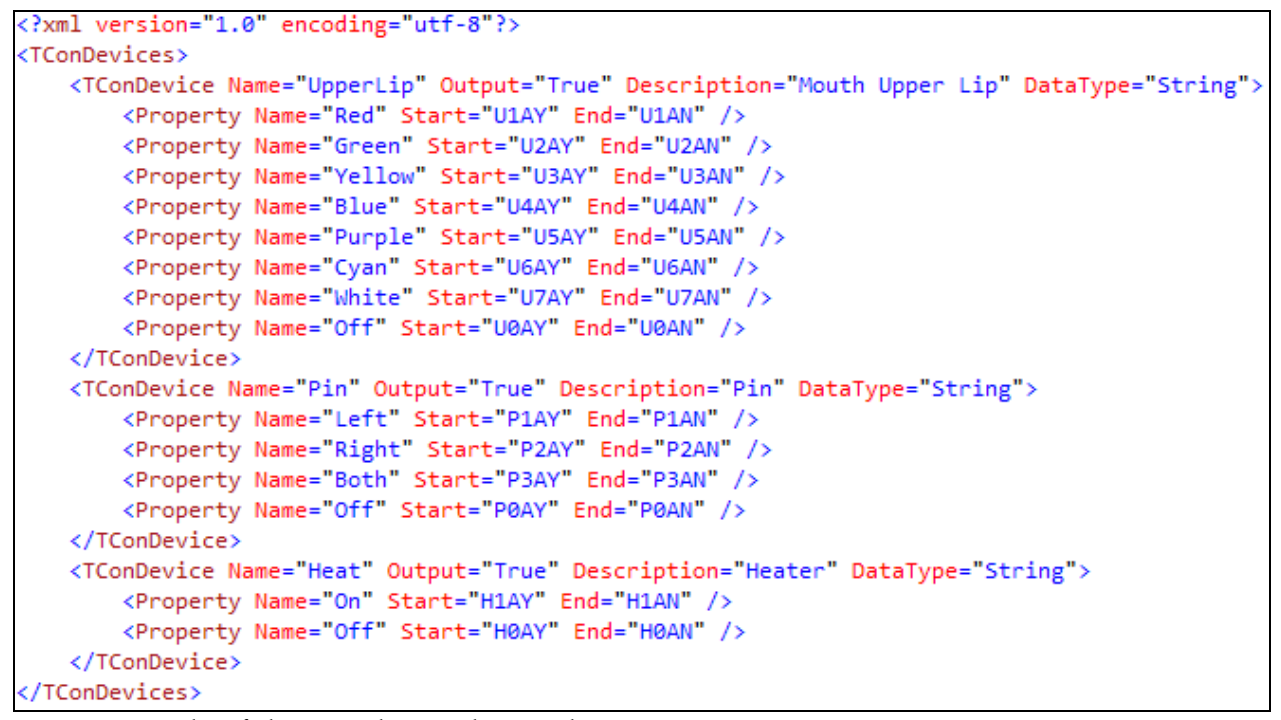

Fig. 2. Example of the TouchCon device description

Figure 2 shows an example of the TouchCon Device XML schema. The root 'TConDevices' can contain multiple 'TConDevice' tags and one 'TConDevice' tag can contain multiple 'Property' tags. To understand the meaning of the example in Figure 2, we can see three actuators are involved in the framework; Upper Lip at line 3, Pin at line 13, and Heat at line 19. And also, we can identify that all three hardwares act as actuators from Output attributes. The values of each Start and End attributes inside the TConDevice tags are the unique commands for hardwares. These commands are totally dependent on the developer's hardwares. Currently, only ASCII strings are allowed to be used as commands.

\subsection{XML Schema for Action Description}

Unlike traditional text-based emoticons, the big changes in multimedia-enabled and hapticembedded emoticons are able to deliver timeline-based actions. For example, a multimediaenabled emoticon can play a small size animation with music. A haptic-embedded emoticon, the next generation of the emoticon, has additional features along with the timeline; a triggered hardware, its duration, and its property to be activated at each moment.

The TouchCon Action is a single element of hardware activation. And TouchCon Library is a bundle of actions. One action describes the device to be activated and its activation time. This is very similar to the music score. In other words, the TouchCon Library schema is the rule to write a score regarding haptic. Table 4 describes the schema of TouchCon Library and Action. 


\begin{tabular}{|l|l|}
\hline Name & Attributes \\
\hline TCons & $\begin{array}{l}\text { (optional) } \\
\text { User: name of the author }\end{array}$ \\
\hline TCon & $\begin{array}{l}\text { (mandatory) } \\
\text { Name: Name of the TouchCon action } \\
\text { (optional) } \\
\text { Image: small icon to display with the haptic action. } \\
\text { Speed: running speed to be executed in the runtime component } \\
\text { Description: information of the TouchCon. }\end{array}$ \\
\hline Action & $\begin{array}{l}\text { (mandatory) } \\
\text { Device: Name of the device to be actuated } \\
\text { StartTime: Start time in millisecond } \\
\text { Duration: Duration time to play in millisecond } \\
\text { (optional) } \\
\text { Property: One of the device command }\end{array}$ \\
\hline
\end{tabular}

Table 4. Description on the haptic library schema

Figure 3 below shows an example of the TouchCon Library schemas in Table 4. According to Table 4, the library here has three levels of depth. We design the schema to have the minimum depth with many attributes, because the XML parser tends to slow down when the depth increases.

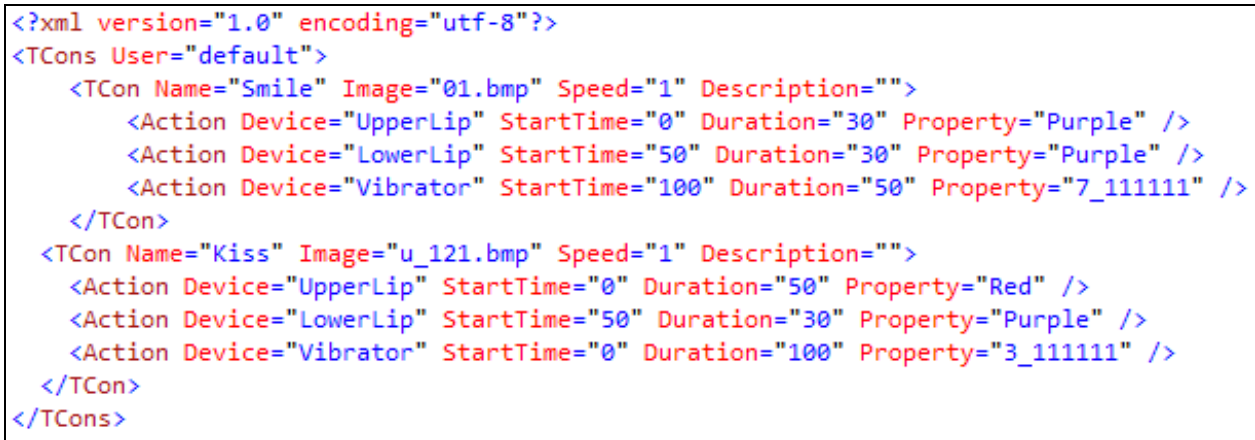

Fig. 3. Example of the haptic device description

In contrast to the TouchCon Device schema, the TouchCon Library one is for users, not for developers. As we can see in Figure 3, one TouchCon Library (TCons) can contain one or more TouchCon Actions (TCon tags). And one TouchCon Action has a list of commands and times.

A single TouchCon Action can be represented as one haptic emoticon. Thus, it can be played on the user's hardware or sent to the peer's one.

\subsection{TouchCon XML Parser}

As all TouchCon-based data are handled and delivered in the XML format, the XML parser is installed in the framework. The TouchCon parser encodes and decodes TouchCon data; 
the TouchCon Library, included actions, sensor specifications, and hardware descriptions. Once the TouchCon parser receives TouchCon Action data, it loads the data in the inmemory database in the FIFO manner. The in-memory database is an array-list so that it is expandable. There are pros and cons to make the in-memory data structure and the XML structure identical. Firstly, the pros; it has to be easy to convert, simple to understand for the user (or the developer), and easy to allocate for very large data. Now, the cons; it tends to cause memory abuse because of the unused TouchCon data, and it leads to the rather long processing time to be allocated into memory. Only two XML schemas were used in our framework, but the implemented TouchCon parser requires to interpret four types of XML data structures; TouchCon Library, TouchCon Action, TouchCon Device, and TouchCon sensor. One reason is that the Library is not just a bundle of actions, but additional information exists. And the other reason is the device and sensors are designed to share the same format for the realization of the bidirectional haptic concept (see Section 3.1), but from the view of the parser, they are not handled in the same way. In short, the two schemas are implemented to four structures.

\section{Haptic Composer}

This chapter introduces the Haptic Editor and the Haptic Resolver. Both aim to enhance the usefulness of the proposed framework. The Haptic Editor is a WYSIWYG editor with attached haptic hardwares. The Haptic Resolver is one of the modules in the TouchCon Core (see Figure 1), which negotiates haptic actuations when two corresponding peers use different hardwares.

\subsection{Haptic Editor}

The Haptic Editor is a timeline-based TouchCon editing tool. Today, many computer-savvy users are familiar with timeline-based multimedia editing tools such as Microsoft MovieMaker or Adobe Flash. Apart from the previous works (Aleven et al. 2006; Mohamad Eid et al. 2008), our Haptic Editor was designed in a WISIWIG manner. Basically, such a tool consists of two core parts; the horizontal part stands for time and the vertical part stands for elements. Timeline is labeled horizontally and elements are arranged vertically. Logically, the timeline and the involved elements are unlimited. Similar to common multimedia editing tools, our system is trigger-based one. Namely, each action is activated after the running time is passed at the designated moment. 


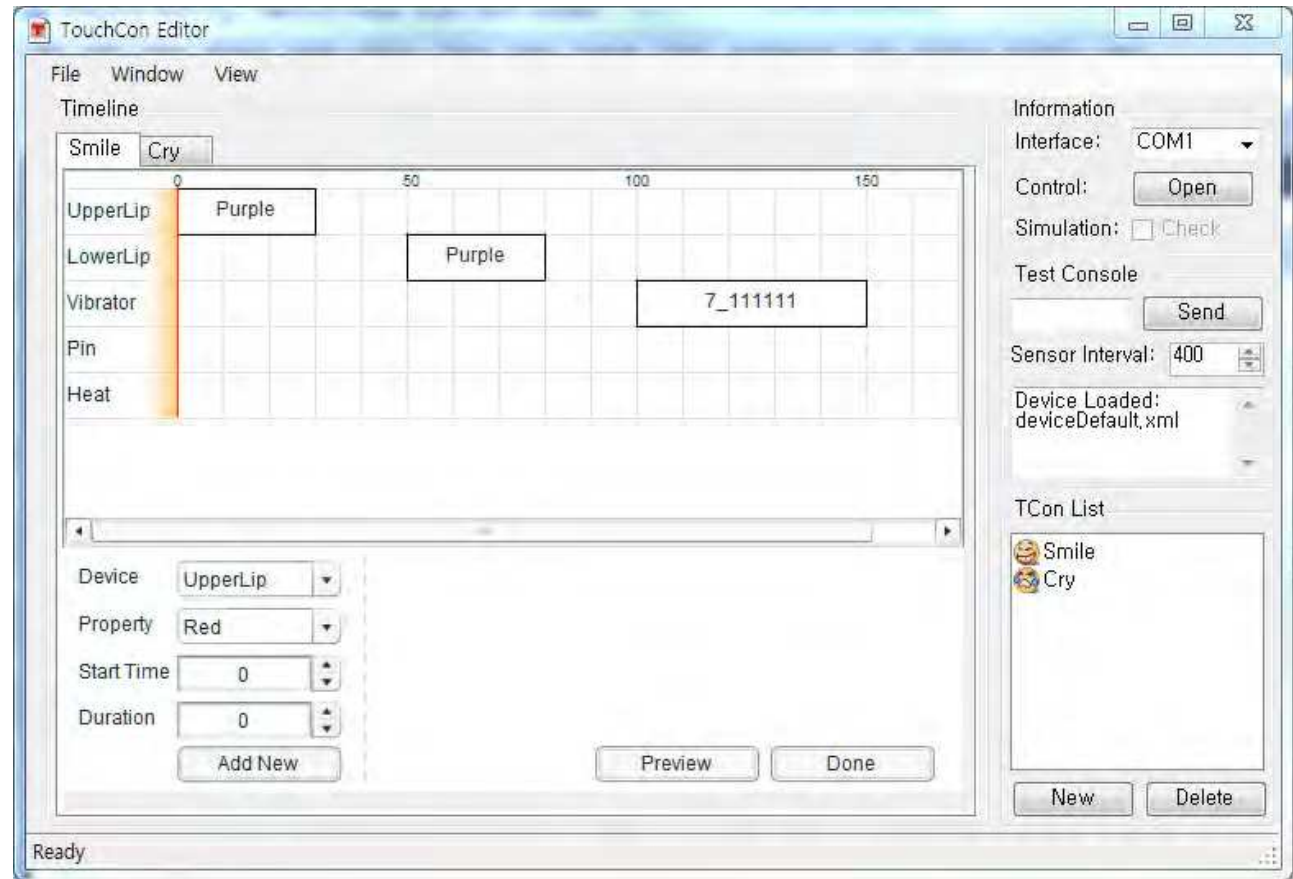

Fig. 4. Haptic Editor

Figure 4 shows a screenshot of the TouchCon Editor. This editor was designed to compose TouchCon Actions and to save them in the TouchCon Library file. The vertical layers indicate available (or controllable) haptic hardwares while the horizontal bars, durations of each action. The text label in the middle of the duration bar is for the property of the hardware. For example as in Figure 4, the label 'Red' indicates the light color of the LED.

The 'Preview' button at the bottom of the window executes (or plays) the current actions and activates the connected hardwares in order to test the composed results. When the user finishes making his/her own TouchCon Actions, the only thing to do is to click the 'Done' button to save the TouchCon haptic actions. Once the button is clicked, a popup window (save dialog) appears in order to incorporate a thumbnail image or additional descriptions. 


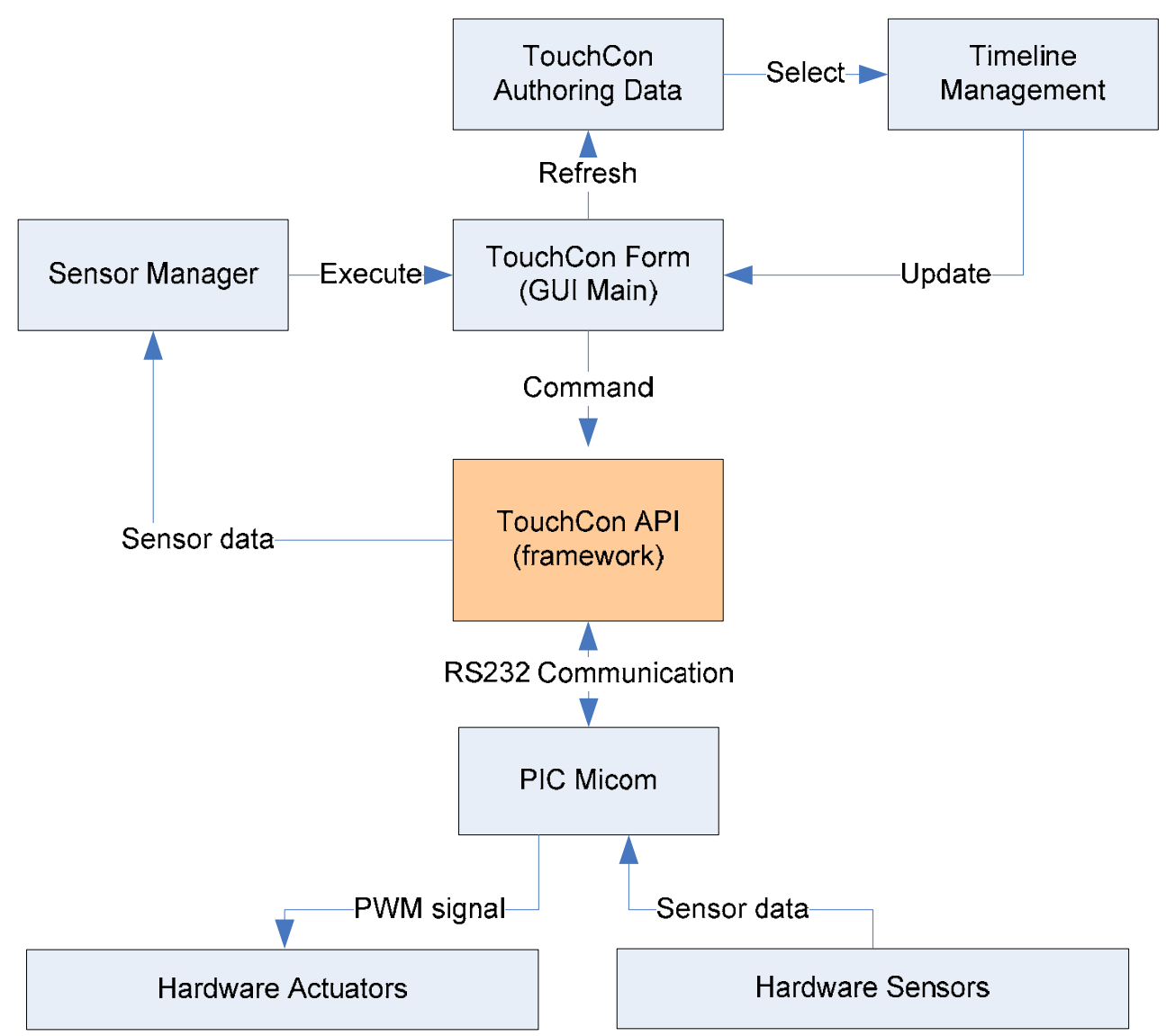

Fig. 5. Architecture of the Haptic Editor

Figure 5 illustrates how the Haptic Editor is constructed. The TouchCon framework communicates with a microcontroller through the RS232 serial port. The RS232 serial port can be replaced by the USB or the Bluetooth interface. The 'PIC Micom' stands for the Microchip ${ }^{\circledR}$ PIC microcontroller. We use an 8 bit microcontroller as the default, but the developer can use any type of microcontroller as far as the developer provides a proper TouchCon Device file.

As we can see in the middle (the orange-colored box), the Haptic Editor also uses the API of the TouchCon framework. The API allows the editor to create, append, remove, and arrange TouchCon Actions. The sensor is handled by the sensor manager. With the sensor data, the sensor manager executes one of three activities; activate user's hardwares, transmit a TouchCon to a peer, or do nothing. The decision along with the value from the sensor has to be defined in the TouchCon Action. Currently, the sensor-related implementation available in our work is only the rule-based decision. For instance, the $0-255$ analog value (or 8 bit resolution) from a microcontroller can be categorized into three ranges and each range has 
its own activity. Generally, $0-30$ is set to 'Do nothing' because such a low intensity value tends to be a noise.

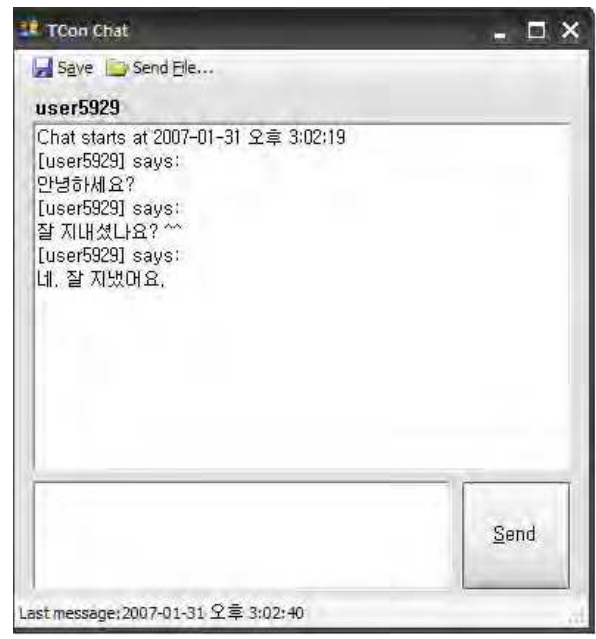

Fig. 6. Instant messenger for testing

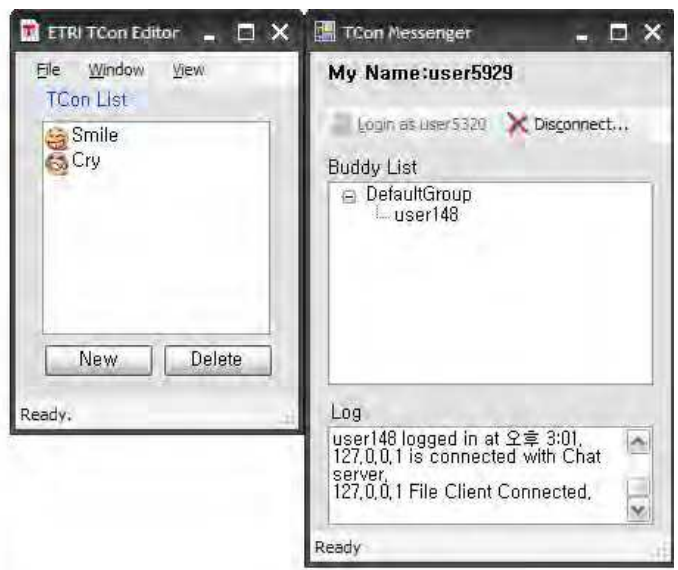

A simple type of an instant messenger is implemented. This program is applied to the demonstration system and used for the evaluation and the survey. The demonstration and its result data are given in section 5.1. In Figure 6, three window-based programs are introduced. The left window is a chat window for the conversation among peers. Users can send text messages, graphical emoticons, or TouchCons. The middle window lists up the available TouchCons. This window is designed to be located nearby the chat window. The user can switch between TouchCon Editor and the list by clicking 'view' button. Namely, the user can easily create his/her own TouchCon while doing chat. Moreover, the messenger automatically adds new TouchCon to the list if the receiver does not have the TouchCon that the peer sends. Finally, the right window is a messenger server that shows available peers on the network. Entire programs are coded in C\# language and run on the Windows XP operating system with the .Net Framework version 2.0.

\subsection{Haptic Resolver}

What happens if a peer sends TouchCons using a cellular phone and the other receives it with a laptop which cannot activate the received TouchCons? To solve this problem, the platform has to resolve this discrepancy and modifies the TouchCons from the sender to the acceptable and similar ones at the receiver.

Next is a simple example of the Haptic Resolver. At first, the magnitude of a TouchCon Action is analyzed. The three attributes to activate haptic actuators are the frequency, the amplitude, and the duration. Based on these, we can represent waveforms or the PWM (Pulse Width Modulation) signals accurately. We found that the waveform is very similar to sound signals. Thus, we utilize this metaphor to convert TouchCons or emoticons to haptic actuator signals through the resolver. Figure 7 shows an example of this conversion. 


\section{: Laugh Cheerfully}

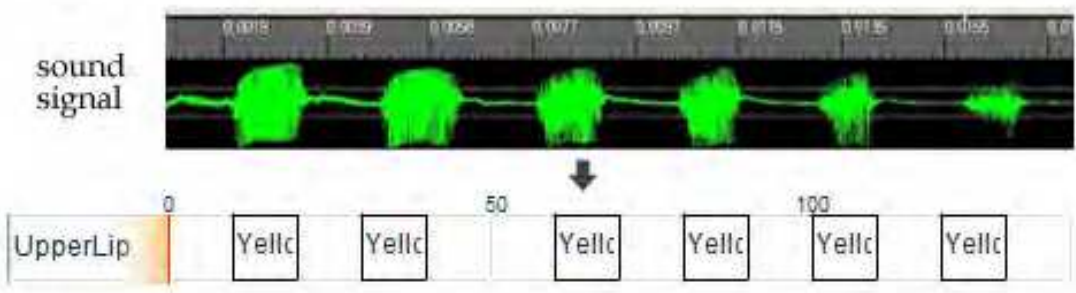

: Cry Bitterly

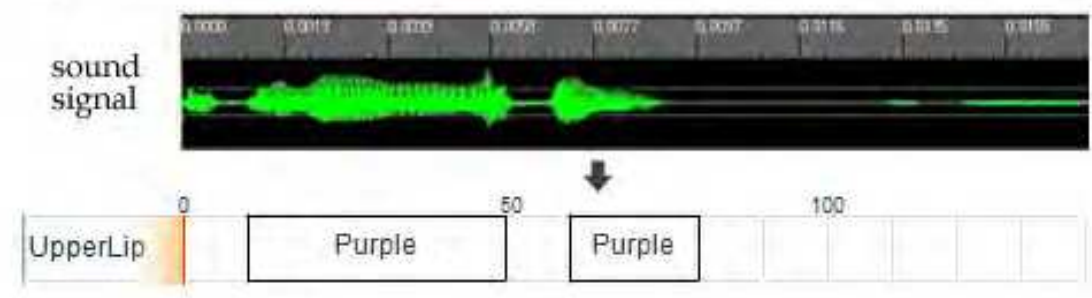

Fig. 7. Sound signals and vibration mapping patterns

The upper part of each box shows recorded sounds of different sensations. During the survey, subjects showed high preferences and high sensational sympathy for more than half of the haptic expressions when the sound mapping is applied. The preference survey results are described in Section 5.

\section{Evaluation}

To evaluate the proposed architecture, several hardware prototypes and software applications are implemented. This chapter introduces how such prototypes and applications work and how they can be utilized to expand the scope of human communications.

\subsection{Implementation of Haptic Testbed for Instant Messenger Environment}

A hardware testbed with various actuators and sensors is implemented. The testbed is designed for the instant messaging environment which is the main target of our system. Industrial designers joined the project and proposed the idea of the palm-rest-shaped silicon forms and a lip-shaped module to make a user feel more humane. The designer wants the user to touch and feel the hardwares like a small pet. For that, the hardwares were covered with the soft-feeling silicon material. In addition, we found that the silicon finish could prevent the user from being injured by embedded heater units. 


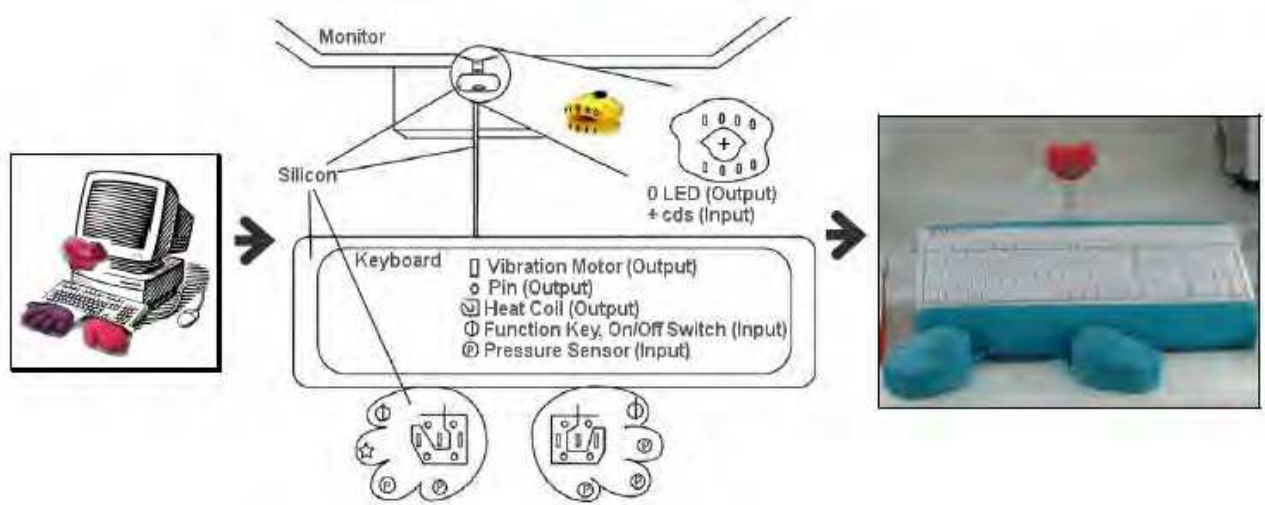

Fig. 8. Design and development process

Figure 8 describes hardware products and their embedded components. At first, a conceptual design was sketched. Then, sensors, actuators, and related circuits were placed in consideration of the hand positions on the products. Later, PCB boards are installed inside the specially designed foams.

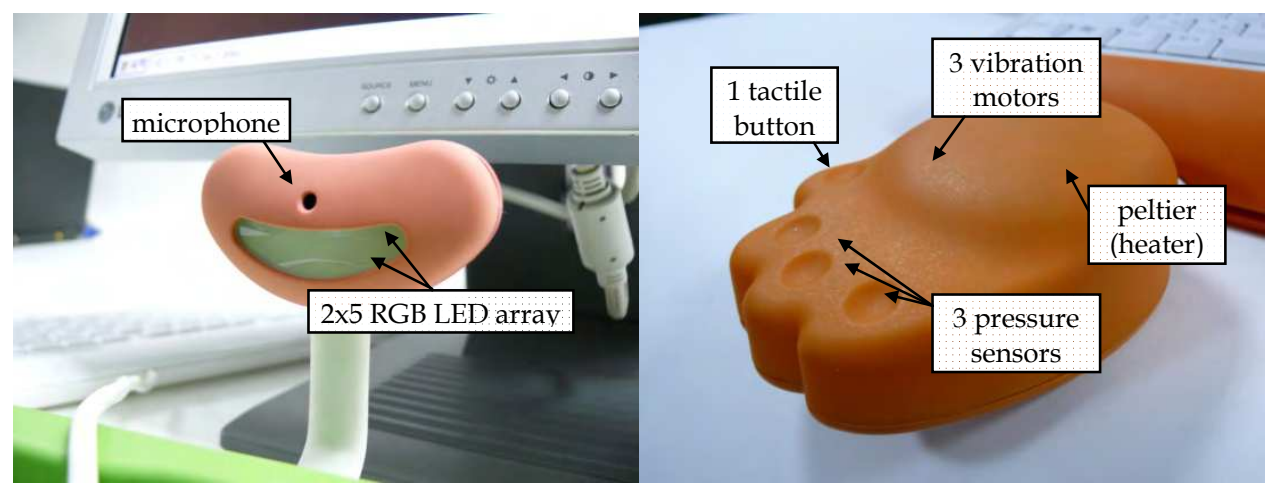

Fig. 9. Actuators and sensors inserted into each hardware part

As can be seen in Figure 9, one animal-foot-shaped palm-rest component has one tactile button, three pressure sensors, three vibration motors and one heater panel. The lip-shaped compartment has ten RGB-color LEDs and one microphone. The microphone can detect the user's touch. Each foot-shaped component is attached to the keyboard using a multiple-wire thick cable. This separated design allows the user to adjust the palm-rest position easily. 


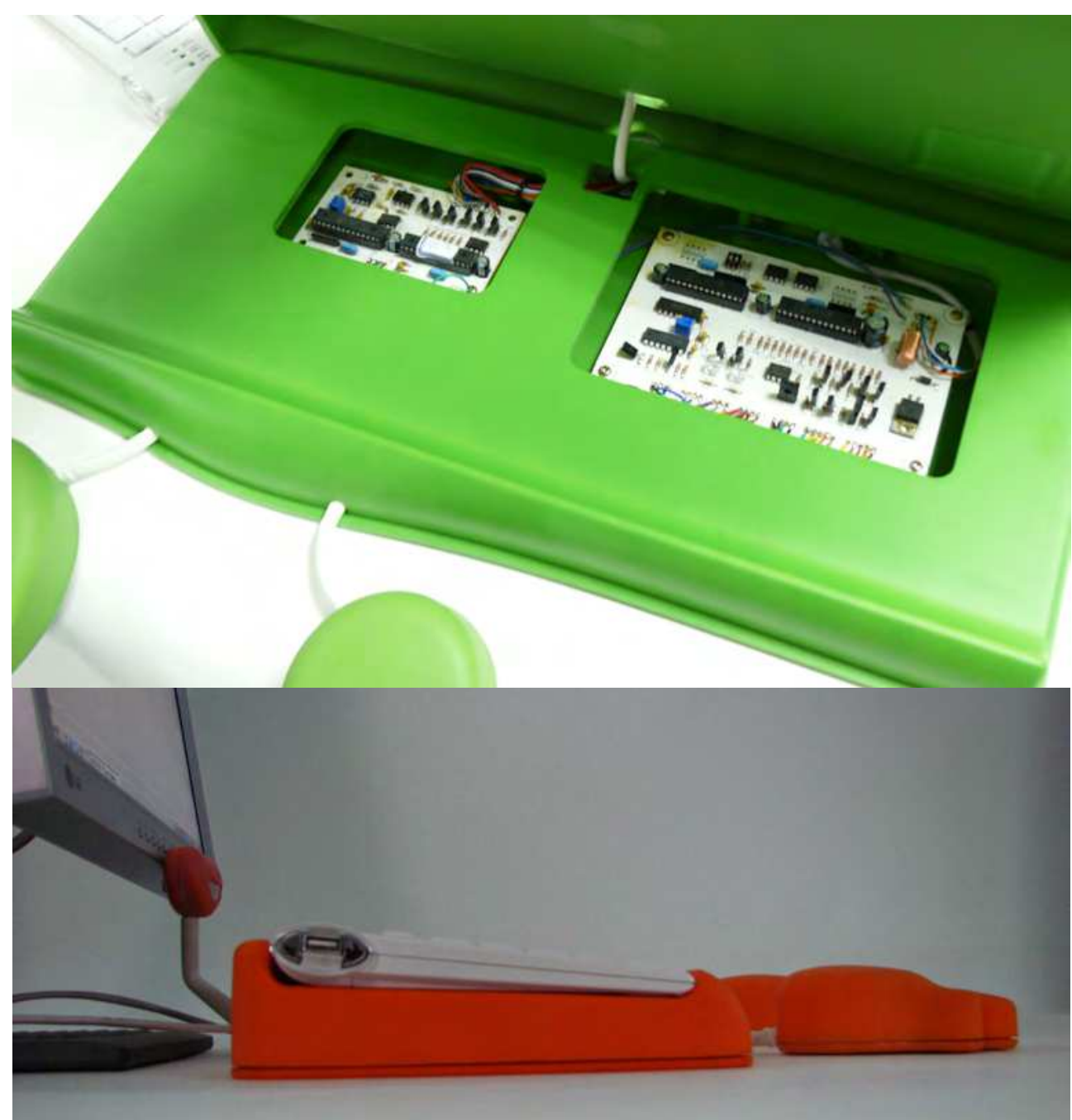

Fig. 10. Controller circuits underneath the keyboard base

Figure 10 shows the controller circuits underneath the keyboard. Thanks to the thin keyboard, i.e., the pentagraph-type keyboard, we can place circuits in the forms seamlessly without losing comfortable typing experiences. The two devices (above and below in Figure 10) are same devices except their color. The left circuit handles the lip-shaped component while the right circuit manages the animal-foot-shaped one. Both circuits use two microcontrollers in order to control the input and the output signal separately. 


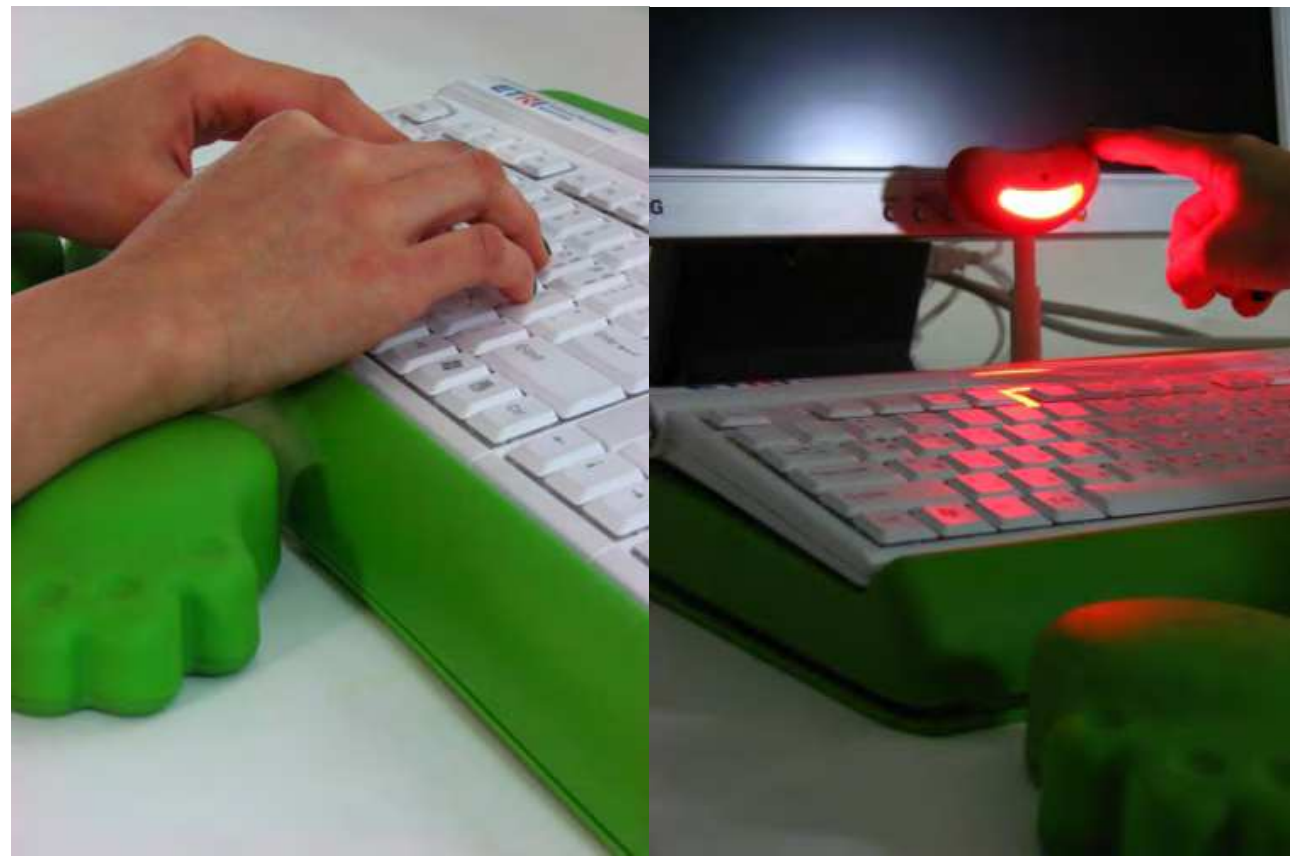

Fig. 11. Usage of prototype hardware

Figure 11 is an example of the hardware usage. The left picture shows how the user can feel the actuation of the vibration motor. The right picture illustrates how the light blinks when the user touches the lip-shaped component.

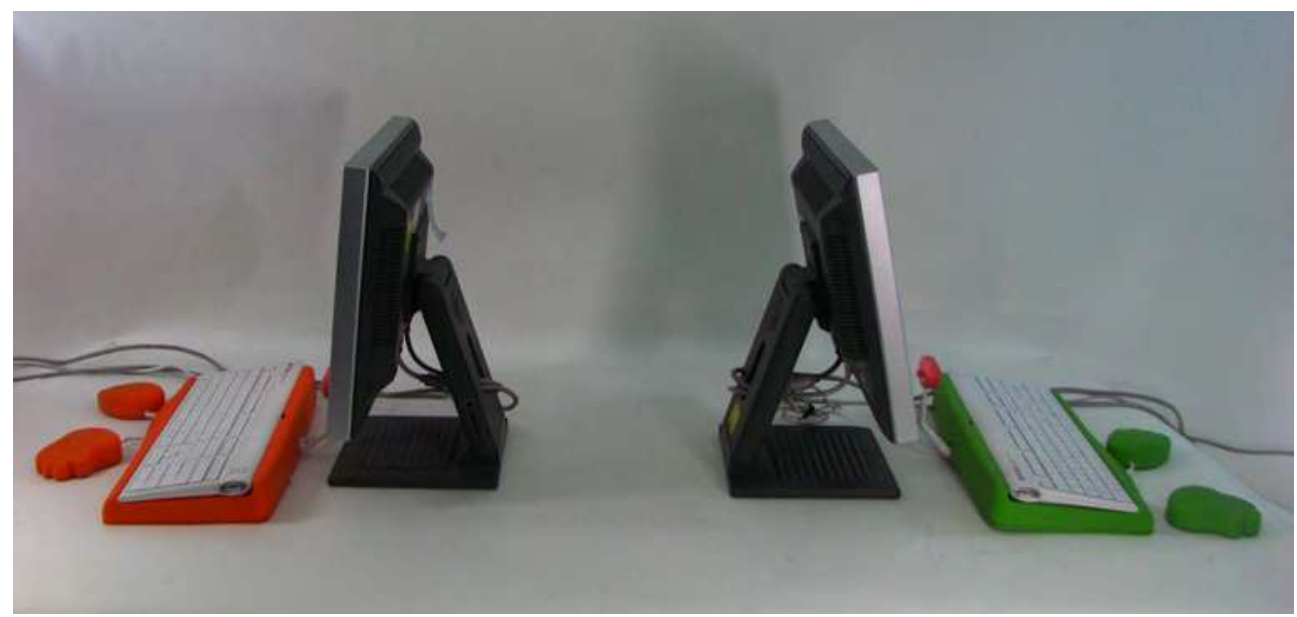

Fig. 12. Demonstration and evaluation setup 
Figure 12 shows a pair of connected computers with our haptic testbed. Total three hardware sets in different colors (orange, green, and blue) were fabricated to survey the user preference. Two of them are used for the survey and the remaining one is for spare. The survey system was demonstrated at the Next Generation Computing Exhibition held in November, 2006, in Korea. During the exhibition, visitors were invited to experience our system and at the same time, the survey was also carried out.

\subsection{User Test}

The objective of the user test is to find out whether haptic expressions are sufficient to make users feel intended emotions. A total of 12 participants (six males and six females) were invited to evaluate TouchCons. Firstly, each TouchCons is presented to them, then they were asked to pick one best-matching emoticon from the list of six, that seemed to serve its purpose best. No prior information about the tactile or visual cues has been provided. Secondly, each participant was asked to evaluate the effectiveness of the TouchCons in representing different types of emotion. The average score was 1 point on a scale from -2 to 2 (five-point Likert scale). Figure 13 shows six selected emoticons and their haptic expressions while Figure 14 shows the two above-mentioned evaluation results.

\begin{tabular}{|c|c|c|c|c|c|c|c|c|}
\hline \multirow{4}{*}{$\begin{array}{l}\text { Emotion } \\
\text { Category }\end{array}$} & \multirow{4}{*}{ Emotion } & \multicolumn{7}{|c|}{ TCON } \\
\hline & & \multirow{3}{*}{$\begin{array}{l}\text { Visual } \\
\text { Exaression }\end{array}$} & \multicolumn{6}{|c|}{ Tactile Expression } \\
\hline & & & \multicolumn{4}{|c|}{ Yibrating Mator } & \multicolumn{2}{|c|}{ Others } \\
\hline & & & $\begin{array}{l}\text { voltage } \\
\left(\begin{array}{l}\text { mintio } 1 \\
\text { max } 2.8)\end{array}\right.\end{array}$ & Duration (ms) & $\begin{array}{l}\text { Delay } \\
(\mathrm{ms})\end{array}$ & $\begin{array}{l}\text { Nuitiber } \\
\text { of } \\
\text { Times }\end{array}$ & $\begin{array}{l}\text { Pit } \\
\text { Actuator } \\
\text { Orvofi }\end{array}$ & $\begin{array}{l}\text { Heat } \\
\text { Display } \\
\text { ONvoFF }\end{array}$ \\
\hline \multirow{2}{*}{ POSIElye } & \multirow{2}{*}{ Gnत } & \multirow{2}{*}{ 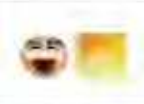 } & \multicolumn{6}{|c|}{ quick, light and regular vibrettory } \\
\hline & & & 19 & 100 & 100 & 5 & off & off \\
\hline \multirow{4}{*}{ Negative } & \multirow{2}{*}{ Cry } & & \multicolumn{6}{|c|}{ slow-moving ybration, night $\rightarrow$ weak $\rightarrow$ mid $-x$ weak stress } \\
\hline & & & $\begin{array}{r}6.8->0.3 \\
->19-8.0 \\
\end{array}$ & \begin{tabular}{|c|}
$500-3700$ \\
$->900->1100$
\end{tabular} & D & 1 & off & off \\
\hline & \multirow{2}{*}{ Anger } & & \multicolumn{6}{|c|}{ one of stong vbration + pir operation } \\
\hline & & & 26 & 200 & 0 & + & an & off \\
\hline \multirow{2}{*}{ Suralise } & \multirow{2}{*}{ Surdrise } & \multirow{2}{*}{69} & \multicolumn{6}{|c|}{ one varation of thick and mid-stress } \\
\hline & & & $1.9-\$ 0.3$ & $800->200$ & $D$ & $T$ & off & alf \\
\hline \multirow[b]{2}{*}{ Winsome } & \multirow{2}{*}{ Kiss } & & \multicolumn{4}{|c|}{ belng strong gradually and continuousy + Heat } & \multicolumn{2}{|c|}{ displav operation } \\
\hline & & & $\begin{array}{c}0.1-210.8 \\
->19->2.8\end{array}$ & \begin{tabular}{c|}
$800->800$ \\
$->1800->1500$
\end{tabular} \mid & D & 1 & on & ori \\
\hline \multirow{2}{*}{$\begin{array}{l}\text { State of } \\
\text { Body }\end{array}$} & \multirow{2}{*}{ Sleepy } & \multirow{2}{*}{-3} & \multicolumn{6}{|c|}{ sow and regular viration } \\
\hline & & & $\begin{array}{c}0.3->1.9 \\
-0.03-1.9\end{array}$ & 1500 & Q & 2 & aff & aff \\
\hline
\end{tabular}

Fig. 13. Selected emoticons and haptic patterns 


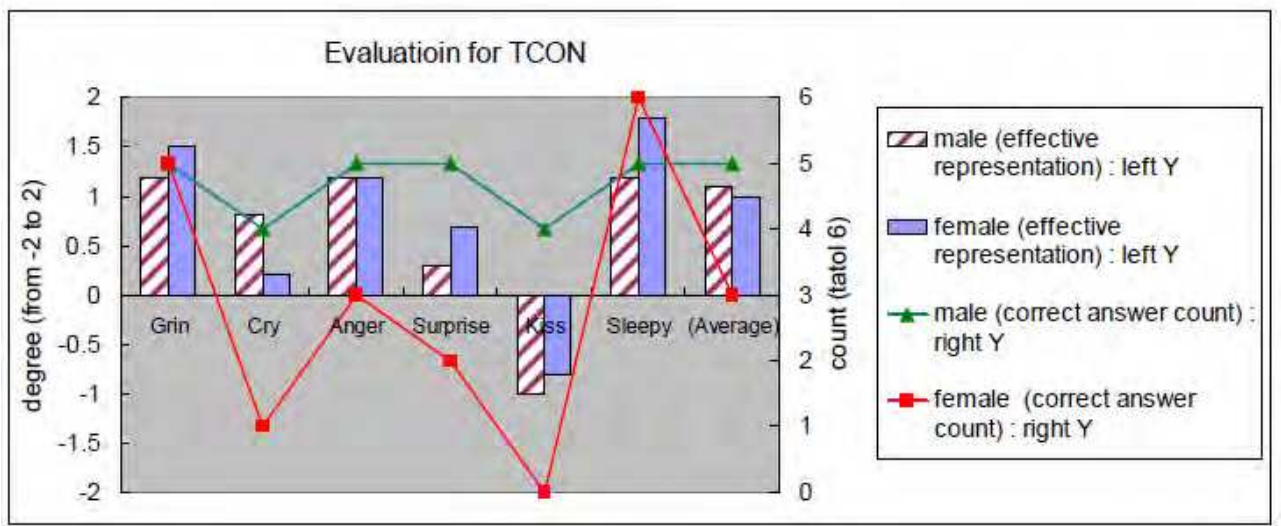

Fig. 14. Evaluation results for TouchCons

In Figure 14, the two lines indicate the first evaluation results (referenced on the right $Y$ axis), and the bars indicate the second evaluation results (referenced on the left $Y$ axis). The results show that the 'Kiss' TouchCon usually failed to give the sensation of kissing, but 'Sleepy' and 'Grinning' were rather successful. Note also that considerable differences exist between female and male users; the former tended to answer with the correct TouchCon less frequently and feel that the TouchCon patterns were less effective than the latter.

Although the TouchCon interface is more complex than that of text emoticons because users have to switch a window focus between the chat and the TouchCon list window, the average number of TouchCons used during each chat reached 14, while that of text emoticons was slightly higher than 17. Finally, a questionnaire survey was conducted after the free experience of the system. The questions included were how enjoyable, emotional, fresh, new, and absorbing the chatting experience was. Respondents were also asked how easy they thought it was to feel the tactile stimulus and how well the pattern chosen suited each type of emotion. Respondents gave the most positive responses on how fresh, new and enjoyable the chat felt ( -2 is the most negative while +2 is the most positive). It was observed that males were more satisfied with the experience than females. Some more additional results can be found in our previous work (Shin et al. 2007; Jung 2008).

\section{Conclusion}

This work was conducted on the combination of two fields, i.e., haptic and social messaging. Haptic is one of the most attention-drawing fields and the biggest buzzwords among nextgeneration users. Haptic is being applied to conventional devices such as the cellular phone and even the door lock. Diverse forms of media such as blogs, social network services, and instant messengers are used to send and receive messages. That is mainly why we focus on the messaging experience, the most frequent communication of the device-mediated conversation.

We propose the integration of sensors and actuators in a single framework in order to make the usage be understood more easily. The specifications to manipulate hardwares require a very light burden to developers; they only need to know the command list which follows 
the TouchCon Device schemas to cooperate their own haptic hardwares with our framework. In conclusion, the haptic communication system proposed in this study enables people to enjoy text messaging with haptic actions and can boost message-based communications among people.

\section{References}

Aleven, V., J. Sewall, B. M. McLaren, and K. R. Koedinger. 2006. Rapid authoring of intelligent tutors for real-world and experimental use. In Advanced Learning Technologies, 2006. Sixth International Conference on, 847-851.

Arnold, K., R. Scheifler, J. Waldo, B. O'Sullivan, and A. Wollrath. 1999. Jini Specification. Addison-Wesley Longman Publishing Co., Inc. Boston, MA, USA.

Baldauf, M., S. Dustdar, and F. Rosenberg. 2007. A survey on context-aware systems. International Journal of Ad Hoc and Ubiquitous Computing 2, no. 4: 263-277.

Bonanni, L., C. Vaucelle, J. Lieberman, and O. Zuckerman. 2006. TapTap: a haptic wearable for asynchronous distributed touch therapy. In Conference on Human Factors in Computing Systems, 580-585. ACM New York, NY, USA.

Botts, M., and A. Robin. 2007. Sensor model language (SensorML). Open Geospatial Consortium Inc., OGC: 07-000.

Brave, S., and A. Dahley. 1997. inTouch: a medium for haptic interpersonal communication. In Conference on Human Factors in Computing Systems, 363-364. ACM New York, NY, USA.

Chang, A., S. O'Modhrain, R. Jacob, E. Gunther, and H. Ishii. 2002. ComTouch: design of a vibrotactile communication device. In Proceedings of the 4th conference on Designing interactive systems: processes, practices, methods, and techniques, 312-320. ACM New York, NY, USA.

Eid, Mohamad, Sheldon Andrews, Atif Alamri, and Abdulmotaleb El Saddik. 2008. HAMLAT: A HAML-Based Authoring Tool for Haptic Application Development. In Haptics: Perception, Devices and Scenarios, 857-866. http://dx.doi.org/10.1007/978-3-540-69057-3_108.

El-Far, F. R., M. Eid, M. Orozco, and A. El Saddik. 2006. Haptic Applications MetaLanguage. In Tenth IEEE International Symposium on Distributed Simulation and RealTime Applications, 2006. DS-RT'06, 261-264.

Immersion Corp, A. 2007. HAPTICS: Improving the Mobile User Experience through Touch. http://www.immersion.com/docs/haptics_mobile-ue_nov07v1.pdf.

Java, A., X. Song, T. Finin, and B. Tseng. 2007. Why we twitter: understanding microblogging usage and communities. In Proceedings of the 9th WebKDD and 1st SNA-KDD 2007 workshop on Web mining and social network analysis, 56-65. ACM New York, NY, USA.

Jung, Chanhee. 2008. Design of Vibro-tactile Patterns for Emotional Expression in Online Environments. Thesis for the degree of Master, Information and Communications University. http:/ /library.kaist.ac.kr/thesisicc/T0001759.pdf.

Kim, Y., Y. Kim, and M. Hahn. 2009. A context-adaptive haptic interaction and its application. In Proceedings of the 3rd International Universal Communication Symposium, 241-244. ACM. 
Norman, Donald A. 2002. The Design of Everyday Things. Basic Books, ISBN 0-465-06710-7, Jackson, TN.

Poupyrev, I., T. Nashida, and M. Okabe. 2007. Actuation and tangible user interfaces: the Vaucanson duck, robots, and shape displays. In Proceedings of the 1st international conference on Tangible and embedded interaction, 212. ACM.

Rovers, A. F., and H. A. Van Essen. 2004. HIM: a framework for haptic instant messaging. In Conference on Human Factors in Computing Systems, 1313-1316. ACM New York, NY, USA.

Rovers, L., and H. A. Van Essen. 2004. Design and evaluation of hapticons for enriched instant messaging. In Proceedings of EuroHaptics, 4: Vol. 4.

Russinovich, M. E., and D. A. Solomon. 2005. Microsoft Windows Internals, Microsoft Windows Server 2003, Windows XP, and Windows 2000. Microsoft Press.

Shin, H., J. Lee, J. Park, Y. Kim, H. Oh, and T. Lee. 2007. A Tactile Emotional Interface for Instant Messenger Chat. Lecture Notes in Computer Science 4558: 166.

Vilhjálmsson, H. H. 2003. Avatar Augmented Online Conversation. Massachusetts Institute of Technology.

Youngjae Kim, Heesook Shin, and Minsoo Hahn. 2009. A bidirectional haptic communication framework and an authoring tool for an instant messenger. In Advanced Communication Technology, 2009. ICACT 2009. 11th International Conference on, 03:2050-2053. Vol. 03. 


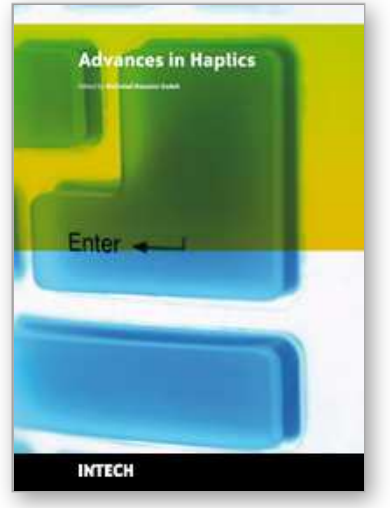

\author{
Advances in Haptics \\ Edited by Mehrdad Hosseini Zadeh
}

ISBN 978-953-307-093-3

Hard cover, 722 pages

Publisher InTech

Published online 01, April, 2010

Published in print edition April, 2010

Haptic interfaces are divided into two main categories: force feedback and tactile. Force feedback interfaces are used to explore and modify remote/virtual objects in three physical dimensions in applications including computer-aided design, computer-assisted surgery, and computer-aided assembly. Tactile interfaces deal with surface properties such as roughness, smoothness, and temperature. Haptic research is intrinsically multidisciplinary, incorporating computer science/engineering, control, robotics, psychophysics, and human motor control. By extending the scope of research in haptics, advances can be achieved in existing applications such as computer-aided design (CAD), tele-surgery, rehabilitation, scientific visualization, robot-assisted surgery, authentication, and graphical user interfaces (GUI), to name a few. Advances in Haptics presents a number of recent contributions to the field of haptics. Authors from around the world present the results of their research on various issues in the field of haptics.

\title{
How to reference
}

In order to correctly reference this scholarly work, feel free to copy and paste the following:

Youngjae Kim and Minsoo Hahn (2010). Expanding the Scope of Instant Messaging with Bidirectional Haptic Communication, Advances in Haptics, Mehrdad Hosseini Zadeh (Ed.), ISBN: 978-953-307-093-3, InTech, Available from: http://www.intechopen.com/books/advances-in-haptics/expanding-the-scope-of-instantmessaging-with-bidirectional-haptic-communication

\section{INTECH}

open science | open minds

\section{InTech Europe}

University Campus STeP Ri

Slavka Krautzeka 83/A

51000 Rijeka, Croatia

Phone: +385 (51) 770447

Fax: +385 (51) 686166

www.intechopen.com

\section{InTech China}

Unit 405, Office Block, Hotel Equatorial Shanghai

No.65, Yan An Road (West), Shanghai, 200040, China 中国上海市延安西路65号上海国际贵都大饭店办公楼405单元

Phone: +86-21-62489820

Fax: $+86-21-62489821$ 
(C) 2010 The Author(s). Licensee IntechOpen. This chapter is distributed under the terms of the Creative Commons Attribution-NonCommercialShareAlike-3.0 License, which permits use, distribution and reproduction for non-commercial purposes, provided the original is properly cited and derivative works building on this content are distributed under the same license. 